\title{
BMJ Open Protocol for a scoping review to identify and map intervention components of existing school-based interventions for the promotion of physical activity and cardiorespiratory fitness among school children aged $6-10$ years old
}

\author{
Berit Brandes (D) , ${ }^{1}$ Heide Busse (D) , ${ }^{1}$ Louisa Sell, ${ }^{1}$ Lara Christianson (D) , \\ Mirko Brandes (10) 1
}

To cite: Brandes B, Busse $\mathrm{H}$, Sell L, et al. Protocol for a scoping review to identify and map intervention components of existing school-based interventions for the promotion of physical activity and cardiorespiratory fitness among school children aged 6-10 years old. BMJ Open 2020;10:e037848. doi:10.1136/ bmjopen-2020-037848

- Prepublication history and additional material for this paper are available online. To view these files, please visit the journal online (http://dx.doi. org/10.1136/bmjopen-2020037848).

Received 18 February 2020

Revised 10 August 2020

Accepted 26 August 2020

\section{A) Check for updates}

(C) Author(s) (or their employer(s)) 2020. Re-use permitted under CC BY-NC. No commercial re-use. See rights and permissions. Published by BMJ.

${ }^{1}$ Prevention and Evaluation, Leibniz Institute for Prevention Research and Epidemiology, BIPS, Bremen, Germany ${ }^{2}$ Clinical Epidemiology, Leibniz Institute for Prevention Research and Epidemiology, BIPS, Bremen, Germany

Correspondence to Berit Brandes;

bbrandes@leibniz-bips.de

\section{ABSTRACT}

Introduction Physical inactivity is known as a leading cause of mortality and tracks from childhood to adulthood. Many types of school-based single-component and multicomponent interventions to promote physical activity (PA) have been undertaken and evaluated, with mixed findings overall. Enlarging the intervention areas beyond the school setting is a promising approach. WHO's Health Promoting School (WHO HPS) framework is a holistic, setting-based approach where health is promoted through the whole school environment with links to other settings such as the home environment and wider community. In this paper, we outline our scoping review protocol to systematically review the published literature from the last 10 years to identify existing school-based interventions to promote PA and cardiorespiratory fitness among children aged 6-10 years old and to map intervention components according to the features of this framework.

Methods and analysis Arksey and 0'Malley's scoping review methodology framework will guide the conduct of this review. We will search Medline, PsycINF0, Cumulative Index to Nursing and Allied Health Literature, Sports Medicine \& Education Index, Education Resources Information Centre and CENTRAL and hand search the reference lists of key studies to identify studies appropriate for inclusion. Any empirical study that evaluated the effectiveness of a school-based intervention promoting PA and/or cardiorespiratory fitness in children aged $6-10$ years old will be included. Two reviewers will independently screen all abstracts and full texts for inclusion. One reviewer will extract general information, study characteristics and intervention contents to classify them according to the features of the WHO HPS framework. Results will be synthesised narratively.

Ethics and dissemination Findings will be disseminated in conference presentations and peerreviewed publications. A condensed version of the results will be made available for the public. Stakeholder meetings will be arranged to discuss and disseminate the findings.

\section{Strengths and limitations of this study}

- This comprehensive overview of existing schoolbased interventions for the promotion of physical activity and fitness in children aged 6-10 years old will follow the Arksey and 0'Malley scoping review methodology and Levac et als methodological enhancement

- This review will identify and map identified interventions and intervention components in relation to WHO Health Promoting School framework

- We will search six electronic databases and article bibliographies to identify relevant studies with no language limits applied.

- We will not provide a synthesis of effectiveness or cost-effectiveness and there will be no formal assessment of study quality.

\section{INTRODUCTION}

According to $\mathrm{WHO}$, physical inactivity is one of the leading risk factors for health and increased mortality. ${ }^{1}$ An active lifestyle is, to a great extent, learnt during the first years of life and tends to persist into adulthood. ${ }^{23}$ Desirable patterns of habitual physical activity (PA) established during the early years and sustained across the lifespan, therefore, may provide the greatest likelihood of impact on mortality and longevity. ${ }^{4}$ Current research shows that, in Europe, few children achieve the recommendation of at least $60 \mathrm{~min}$ of moderate to vigorous PA per day. ${ }^{5}$ Furthermore, the gradual decline in PA between childhood and adolescents is a robust epidemiological phenomenon. ${ }^{6-8}$ Social, environmental and biological factors are likely to contribute to such a marked trend. ${ }^{9}$ 
PA is an overarching term that consists of many structured and unstructured forms within school and out-ofschool-time contexts, including organised sport, physical education, outdoor recreation, motor skill development programmes and active transportation such as biking and walking. Children's PA behaviours are influenced by many sectors of society, including families, communities, schools, healthcare providers and the media and each of these sectors plays an important role in improving PA behaviours of young persons. ${ }^{10}$ As children spend a large proportion of their waking day at school, schools are considered a key setting to promote healthy behaviours such as PA. ${ }^{11}$ There is evidence that about $50 \%$ of children's total PA occurs during school hours. ${ }^{12}$ Consequently, many interventions have been developed and tested with the aim of increasing children's and adolescents' PA within the school setting. However, many types of school-based single-component and multicomponent interventions at different levels have been undertaken and evaluated, with mixed findings overall. ${ }^{13-16}$ Systematic reviews of the field have shown that single-component interventions at the individual level such as classroombased PA interventions were not effective overall. ${ }^{15}$ Some trials reported positive effects during school hours (eg, physical education-based interventions with relatively simple modifications) but did not increase out-of-class PA. ${ }^{17}$ Also, Love et $a l^{18}$ reported in their meta-analysis of cluster randomised controlled trials with accelerometerassessed activity that current school-based efforts did not positively impact young people's PA across the full day. In other words, current evidence suggests that focusing on activities within one setting only is unlikely to make substantial changes to population levels of and inequities in physical inactivity in children.

There is evidence that interventions promoting PA and fitness are promising if they are using WHO's Health Promoting School (WHO HPS) approach by targeting the formal health curriculum, the ethos and environment of the school, and engaging families and communities. ${ }^{11}$ The HPS approach is a holistic, settingbased approach for health promotion in schools, whereby health is promoted through the whole school environment with links to families, the community and health services. ${ }^{11}$ The health promoting school framework by WHO emphasises schools as key settings for healthy living, learning and working and is based on a significant shift in WHO public health policy, in the late 1980 s, from a focus on individual behaviour to recognition of the wider social, political and environmental influences on health. ${ }^{19}$ The six key features of the WHO HPS are: (1) school health policies, (2) physical school environment, (3) social school environment, (4) health skills and education, (5) links with parents and community and (6) Access to (school) health services. ${ }^{20}$

To obtain an overview of existing interventions, this study aims to identify and then map existing school-based interventions for the promotion of PA and/or cardiorespiratory fitness among children aged 6-10 years old according to the features of the WHO HPS framework. This overview will provide a starting point for the development of multilevel interventions that combine promising intervention components, which have mostly been analysed separately. Our wider aim is to provide knowledge for enlarging the intervention areas beyond the school setting, for example, to active transportation and afterschool interventions, and for combining these interventions by using the existing literature of the field from the last 10 years.

\section{STUDY RATIONALE}

Schools remain a promising setting to promote healthy behaviours, such as PA, as they can ensure that a majority of children and young people are reached and as they have potential to ensure that intervention components extent to families, peers and the wider community. Although for traditional activities, whereby health is promoted through 'health education' in the curriculum, there is little evidence to demonstrate that such approaches can effect sustainable behavioural change in the long term, multilevel multicomponent interventions that included changes to the formal health curriculum, changes to the ethos and environment of the school, and links with families and/or communities were deemed promising. ${ }^{11}$ There is no overview of the extent to which school-based interventions to promote PA and cardiorespiratory fitness from the last decade have used a holistic approach and in how far existing interventions have addressed the key features of the WHO HPS framework.

\section{STUDY OBJECTIVES}

The purpose of this scoping review is to identify, describe and map school-based interventions to promote PA and cardiorespiratory fitness in children aged 6-10 years old from the last 10 years. In this context, we define schoolbased interventions as interventions that were distributed mainly in the school setting, whereas the target of performing PAs can refer to the time children spent in school as well as to the time children spent outside of the school. The specific objectives of our scoping review are:

1. To identify single-component and multicomponent school-based interventions for the promotion of PA and cardiorespiratory fitness in the children aged 6-10 years old.

2. To map identified interventions along WHO HPS framework.

3. To provide an overview about what kind of intervention components seem to work.

\section{METHODS AND ANALYSIS}

\section{Theoretical frameworks}

While there is no strict definition of a Health Promoting School, this scoping review will use the six-point definition 


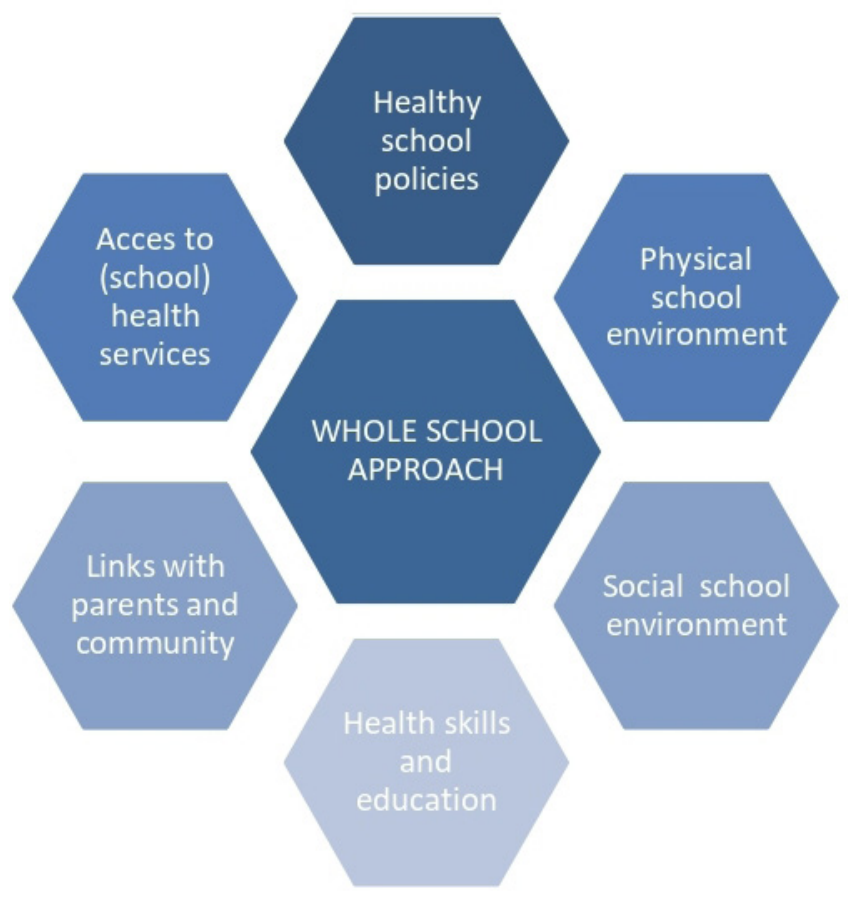

Figure 1 Health promoting schools framework. Adapted from $\mathrm{WHO}^{20}$

of the HPS framework provided by the WHO, ${ }^{20}$ which is shown in figure 1 . Briefly, according to the six key features of the framework, a HealthPromoting School engages teachers, students, parents, community leaders and organisations linked to the school in efforts to promote health. Furthermore a safe and healthy environment, skills-based health education and access to health services are provided. This can be complemented through health promoting policies and practices and through the participation in community health projects. ${ }^{20}$ We are aware that school-based PA promoting interventions rarely cover all of the six features. But we are highly interested in grouping intervention components according to the aforementioned features. Likewise, we will group the following examples as follows: In this scoping review 'School health policies' are seen as whole-school approaches that clearly define documents or accepted practices that promote PA or reduce sedentary activities within the school setting, for example, by proactively promoting an active commute to school or by a ban on the use of electronic devices. Examples for changes to the "physical school environment' include the provision of a safe and PA-promoting environment, for example, outdoor classrooms, playground markings and sports equipment provided within the school setting. Skills-based health education refers to both the formal and informal curriculum and aims at teaching PA-related knowledge, experiences and fundamental movement skills, for example, increased numbers of physical education lessons within the school curriculum or curriculum-focused active breaks (short bouts of PA that include curriculum content) within the classroom. We define PA-related intervention components that aim at changing the 'Social school environment' as whole-school approaches aimed at changing the ethos and norms surrounding PA within the whole school and thus also influence people beyond the target group, such as teachers or other students. Examples for this are activities such as teacher training on the promotion of active breaks or school-wide posters or campaigns promoting PA. Intervention components that, for instance, teach school nurses in PA-related problems are grouped into the category 'Access to (school) health services'. Lastly, any link to parents (eg, workshops with family members or PA-related homework that can only be accomplished by the children with the help of their parents) or any link to the community (eg, after-school programmes, collaborations with sports clubs or organisations, are classified as 'Links with parents and community'. We will map all PA-related intervention components of interventions from the last 10 years using this framework.

\section{Protocol design}

This study follows the Arksey and O'Malley ${ }^{21}$ scoping review methodology, and Levac et al $\mathrm{s}^{22}$ methodological enhancement. According to this methodology, there are five different stages in undertaking a scoping review: (1) identifying the research question(RQs); (2) identifying potentially relevant studies; (3) selecting eligible studies; (4) charting the data; (5) collating, summarising and reporting the results. The Preferred Reporting Items for Systematic Reviews and Meta-Analyses Protocols statement ${ }^{23}$ has been followed (as far as relevant for a scoping review) to verify the structure and content of this protocol (the checklist is available as an online supplemental appendix A). Patients and the public will not be involved in the development of the RQ and the design of the study.

\section{Stage 1: identifying the RQs}

Our RQs were developed and refined in a collaborative effort of the research team (BB, HB and $\mathrm{MB}$ ). In a first step, the literature was screened for existing systematic reviews on school-based and community-based PA interventions targeting children aged 6-10 years old from the last decade. In a second step, we screened the literature for a theoretical framework to be used to map current PA intervention components inside and outside school. This review will, therefore, be guided by the main broad (RQ1) 'What types of school-based interventions for the promotion of PA and cardiorespiratory fitness among school children aged 6-10 years old have been reported from 2010 until 2020?'

Furthermore, two secondary RQs will be used to guide this review: (RQ2) 'Which specific features of the WHO HPS framework are addressed in the identified interventions?' and (RQ3) 'Which specific features of the WHO HPS framework in school-based interventions for the promotion of PA and fitness among school children aged 6-10 years old seem to work?'. Besides answering the main RQs, our thorough data extraction will consider other important factors such as sex-specific tailoring in 


\begin{tabular}{|c|c|c|}
\hline & Search themes & Search terms \\
\hline 1 & Physical activity & $\begin{array}{l}\text { Sport, exercise, fitness, athletic, physical activity, physical education, physical exertion, training, } \\
\text { physical fitness }\end{array}$ \\
\hline 2 & Setting & $\begin{array}{l}\text { Elementary school, entry-level school, grade school, grammar school, preparatory school, primary } \\
\text { school, elementary education, primary education }\end{array}$ \\
\hline 3 & Population & Boy, child, girl, kid, minor, preteen, youth, paediatric \\
\hline 4 & Intervention & $\begin{array}{l}\text { School-based promotion, programme, intervention, initiative, strategy, campaign, school health } \\
\text { services, health promotion }\end{array}$ \\
\hline
\end{tabular}

Consideration of American/British English and pluralisation.

the identified intervention approaches, country-specific differences in the implementation and impact and implementation issues (eg, use of internal and/or external providers).

\section{Stage 2: identifying relevant studies-search terms and inclusion/exclusion criteria}

The search strategy will consist of a combination of keywords and controlled vocabulary. Based on preliminary searches, we will combine the following four search themes with Boolean operators (table 1).

The search query will be developed in Medline and adapted to other databases in collaboration with an experienced research librarian and coauthor. The databases Medline, PsycINFO, Cumulative Index to Nursing and Allied Health Literature, Sports Medicine \& Education Index, Education Resources Information Centre and CENTRAL will be searched. The reference lists of the included studies will be hand searched to identify additional relevant articles.

The currently proposed initial search strategy is shown in online supplemental appendix B. The search will be limited to studies published since 2010. No language limit will be applied. Search results will be downloaded and deduplicated using EndNote software and imported into COVIDENCE $^{24}$ for further review and screening.

The inclusion criteria defined by population, intervention, comparator and outcomes are shown in table 2. Articles will be included if they are empirical studies that evaluated the effectiveness of a school-based intervention promoting PA and/or cardiorespiratory fitness in children aged 6-10 years old. Studies that measured at least one PA and/or cardiorespiratory fitness outcome will be included. Any types of PA measures that include information about the frequency and intensity of an activity will be included. For example, the question 'How often did your child go to school by bike during the last week?' contains information about both dimensions ("to bicycle=at least moderate intensity' and ' 5 times per week'). The duration, rate or proportion of PA (time spent engaged in moderate-to-vigorous PA, light PA, total PA and sedentary behaviour), measured as the total minutes per hour or day or week, either reported through self-report (eg, time spent watching television (TV)/computer/smartphone/ digital versatile disc (DVD)) or through the use of accelerometers during either school or non-school time (or both) will also be included. We will include measures of cardiorespiratory fitness, measured directly (such as $\mathrm{VO}_{2} \max$, heart rate, peak performance) or indirectly (such as shuttle run test, step test, yoyo test, 6 min run).

Studies will be excluded if no intervention component was delivered via school. Studies will also be excluded if they did not apply a controlled pretest/post-test design. Eligible comparator interventions will either receive usual practice or no intervention. Generally, the comparison will be against standard, currently existing physical education programmes in schools.

\section{Stage 3: study selection}

A two-part study selection process will be used: (1) a title and abstract review and (2) full-text review. Inter-rater reliability will be calculated for both stages using Cohen's $\kappa$ statistic. ${ }^{25}$ In the first step, two reviewers will separately review the citations yielded from the search to determine the eligibility based on the defined inclusion and exclusion criteria. To confirm their robustness, the inclusion and

Table 2 Population, intervention, comparator, outcomes

\begin{tabular}{|c|c|}
\hline Population & School children aged $6-10$ years old \\
\hline Intervention & To promote physical activity and/or cardiorespiratory fitness and/or to prevent physical inactivity \\
\hline Comparator & standard, currently existing physical education programmes in schools \\
\hline Outcomes & $\begin{array}{l}\text { Any types of physical activity measures that include information about the frequency and intensity of an activity } \\
\text { will be included (eg, steps per day, amount of time spent in moderate-to-vigorous physical activity, total physical } \\
\text { activity, TV viewing) and/or any cardiorespiratory fitness outcome (eg, } \mathrm{VO}_{2} \text { max, heart rate, peak performance). }\end{array}$ \\
\hline
\end{tabular}

TV, television. 
exclusion criteria will be tested on a sample of abstracts before conducting the actual search to help capture any studies that may be relevant to our RQ. Disagreements between the two authors will be resolved by discussion, with involvement of a third author when necessary. In the second step, two reviewers will independently evaluate the full-text articles to decide if they meet the inclusion/ exclusion criteria. In case of any disagreement about inclusion, full-text articles will be reviewed again by both reviewers and if an agreement cannot be reached, this will be resolved by a consolidation with a third reviewer.

\section{Stage 4: charting the data}

The fourth stage of Arksey and O'Malley ${ }^{21}$ scoping review methodology is the charting of the data of the selected articles. A data extraction form will be developed by the research team to confirm study relevance and to extract study characteristics. Study characteristics to be extracted will include: author(s), publication year, name of trial, study design, country, study population characteristics (eg, age range and sex), total sample size, intervention setting, provider (eg, internal/external), theoretical framework, intervention duration, type of comparator, PA and/or cardiorespiratory fitness outcomes, intervention components, features of the WHO HPS framework that are addressed, and the author's conclusions on effectiveness on PA and/or cardiorespiratory fitness outcomes for the total sample as well as for subgroups where applicable (eg, results of sex stratified analyses). The data extraction will be subjected to a test by both reviewers separately extracting the data from a sample of the included articles. Data extraction will then be conducted by one reviewer. A second reviewer will independently extract data of $10 \%$ of the included studies and results of both reviewers will be compared and discussed within the research team.

\section{Stage 5: collating, summarising and reporting the results}

Findings of this scoping review will provide an overview of the research rather than an assessment of the quality of individual studies. According to RQ2, the intervention contents identified and extracted will be coded based on the six-point definition of the WHO HPS framework as discussed above. From this, a list of promising interventions and intervention contents for further dissemination will be presented. Findings will be discussed in terms of what kind of intervention components seem to work (RQ3).

\section{Limitations}

We limit our search to the last ten years of the publication date. Given the time delay between running a study and its publication, our strategy should cover all interventions which have been implemented and scientifically evaluated within the last 12 years. However, we will not discover interventions that have not been reported on prior to 2009. Moreover, many interventions are published in grey literature, which is also not covered by our review. As common within scoping reviews, we do not assess study quality. However, we hope that our review is suitable to identify any potential intervention for promoting PA and/or cardiorespiratory fitness in children aged 6-10 years old, and that interested readers may easily apply their specific requirements for judging study quality to the indicated studies.

\section{ETHICS AND DISSEMINATION}

Formal ethical approval for the study is not needed as no primary data is collected. The results from this scoping review will guide the next phase of a research programme that will lead to a development of a set of interventions promoting PA and cardiorespiratory fitness via primary schools, the ACTIvity PROmotion via Schools (ACTIPROS) research project. The aim of ACTIPROS is to facilitate ACTIvity PROmotion via Schools by developing a toolbox of intervention components that promote PA and cardiorespiratory fitness in school children aged $6-10$ years old. The set of promising interventions gathered from this scoping review will be disseminated in the toolbox. Our research team will use a participative approach involving national stakeholders to guide the research objectives, the development of the toolbox and the pilot testing.

\section{Twitter Mirko Brandes @MirkoBrandes}

Contributors $\mathrm{BB}, \mathrm{HB}$ and $\mathrm{MB}$ conceived the idea, developed the research question and study methods and contributed meaningfully to the drafting and approved the final manuscript. LS and LC aided in developing the research question and study methods and approved the final manuscript.

Funding This work was supported by the Federal Ministry of Health, Germany, grant number 1504/54401.

Disclaimer The funders had no role in defining the scope of the review and have not been involved in the detailed protocol development presented in this paper.

Competing interests None declared.

Patient and public involvement Patients and/or the public were not involved in the design, or conduct, or reporting, or dissemination plans of this research.

Patient consent for publication Not required.

Provenance and peer review Not commissioned; externally peer reviewed.

Open access This is an open access article distributed in accordance with the Creative Commons Attribution Non Commercial (CC BY-NC 4.0) license, which permits others to distribute, remix, adapt, build upon this work non-commercially, and license their derivative works on different terms, provided the original work is properly cited, appropriate credit is given, any changes made indicated, and the use is non-commercial. See: http://creativecommons.org/licenses/by-nc/4.0/.

\section{ORCID iDs}

Berit Brandes http://orcid.org/0000-0002-9313-6593

Heide Busse http://orcid.org/0000-0001-6043-9072

Lara Christianson http://orcid.org/0000-0002-7780-255X

Mirko Brandes http://orcid.org/0000-0003-2926-4758

\section{REFERENCES}

1 WHO. Global health risks : mortality and burden of disease attributable to selected major risks. Geneva: World Health Organization, 2009.

2 Cleland V, Dwyer T, Venn A. Which domains of childhood physical activity predict physical activity in adulthood? A 20-year prospective tracking study. Br J Sports Med 2012;46:595-602. 
3 Telama R, Yang X, Leskinen E, et al. Tracking of physical activity from early childhood through youth into adulthood. Med Sci Sports Exerc 2014;46:955-62.

4 Paffenbarger RS, Hyde RT, Wing AL, et al. Physical activity, allcause mortality, and longevity of college alumni. $N$ Engl J Med 1986;314:605-13.

5 Konstabel K, Veidebaum T, Verbestel V, et al. Objectively measured physical activity in European children: the IDEFICS study. Int $J$ Obes 2014;38 Suppl 2:S135-43.

6 Van Hecke L, Loyen A, Verloigne M, et al. Variation in population levels of physical activity in European children and adolescents according to cross-European studies: a systematic literature review within DEDIPAC. Int J Behav Nutr Phys Act 2016;13:70.

7 Telama R, Yang X. Decline of physical activity from youth to young adulthood in Finland. Med Sci Sports Exerc 2000;32:1617-22.

8 van Mechelen W, Twisk JW, Post GB, et al. Physical activity of young people: the Amsterdam longitudinal growth and health study. Med Sci Sports Exerc 2000;32:1610-6.

9 Hills AP, King NA, Armstrong TP. The contribution of physical activity and sedentary behaviours to the growth and development of children and adolescents: implications for overweight and obesity. Sports Med 2007;37:533-45.

10 Centers for Disease Control and Prevention (CDC). School health guidelines to promote healthy eating and physical activity. MMWR Recomm Rep 2011;60:1-76.

11 Langford R, Bonell CP, Jones HE, et al. The who health promoting school framework for improving the health and well-being of students and their academic achievement. Cochrane Database Syst Rev 2014;4:Cd008958.

12 Sprengeler O, Wirsik N, Hebestreit A, et al. Domain-Specific selfreported and objectively measured physical activity in children. Int $J$ Environ Res Public Health 2017;14:ijerph14030242.

13 Kriemler S, Meyer U, Martin E, et al. Effect of school-based interventions on physical activity and fitness in children and adolescents: a review of reviews and systematic update. $\mathrm{Br} J$ Sports Med 2011;45:923-30.
14 Mura G, Vellante M, Nardi AE, et al. Effects of school-based physical activity interventions on cognition and academic achievement: a systematic review. CNS Neurol Disord Drug Targets 2015;14:1194-208

15 Watson A, Timperio A, Brown $\mathrm{H}$, et al. Effect of classroom-based physical activity interventions on academic and physical activity outcomes: a systematic review and meta-analysis. Int J Behav Nutr Phys Act 2017;14:114.

16 Dobbins M, Husson H, DeCorby K, et al. School-Based physical activity programs for promoting physical activity and fitness in children and adolescents aged 6 to 18. Cochrane Database Syst Rev 2013;2013:Cd007651.

17 Slingerland M, Borghouts L. Direct and indirect influence of physical education-based interventions on physical activity: a review. J Phys Act Health 2011:8:866-78.

18 Love R, Adams J, van Sluijs EMF. Are school-based physical activity interventions effective and equitable? A meta-analysis of cluster randomized controlled trials with accelerometer-assessed activity. Obes Rev 2019;20:859-70.

19 Ottawa charter for health promotion. Can J Public Health 1986;77:405-05.

20 World Health Organization. Local Action Creating Health Promoting Schools, 2017. Available: https://www.who.int/school_youth health/media/en/sch_local_action_en.pdf?ua=1 [Accessed 10 Feb 2020].

21 Arksey H, O'Malley L. Scoping studies: towards a methodological framework. Int J Soc Res Methodol 2005;8:19-32.

22 Levac D, Colquhoun H, O'Brien KK. Scoping studies: advancing the methodology. Implement Sci 2010;5:69.

23 Shamseer L, Moher D, Clarke M, et al. Preferred reporting items for systematic review and meta-analysis protocols (PRISMA-P) 2015 elaboration and explanation. BMJ 2015;350:g7647.

24 Covidence. systematic review software [program]. Melbourne, Australia, 2019

25 Cohen J. A coefficient of agreement for nominal scales. Educ Psychol Meas 1960;20:37-46. 